\title{
The use of iloprost in early pregnancy in patients with pulmonary arterial hypertension
}

\author{
C.A. Elliot* ${ }^{*+}$, P. Stewart" ${ }^{*}$, V.J. Webster", G.H. Mills", S.P. Hutchinson", \\ E.S. Howarth ${ }^{\S}$, F.A. Bu’Lock ${ }^{\ddagger}$, R.A. Lawson*, I.J. Armstrong* and D.G. Kiely*
}

ABSTRACT: In patients with pulmonary hypertension, pregnancy is associated with a high risk of maternal death. Such patients are counselled to avoid pregnancy, or if it occurs, are offered early interruption. Some patients, however, decide to continue with their pregnancy and others may present with symptoms for the first time whilst pregnant. Pulmonary vasodilator therapy provides a treatment option for these high-risk patients.

The present study describes three patients with pulmonary arterial hypertension of various aetiologies who were treated with the prostacyclin analogue iloprost during pregnancy, and the post-partum period.

Nebulised iloprost commenced as early as 8 weeks of gestation and patients were admitted to hospital between 24-36 weeks of gestation. All pregnancies were completed with a duration of between 25-36 weeks and all deliveries were by caesarean section under local anaesthetic. All patients delivered children free from congenital abnormalities, and there was no post-partum maternal or infant mortality.

In conclusion, although pregnancy is strongly advised against in those with pulmonary hypertension, the current authors have achieved a successful outcome for mother and foetus with a multidisciplinary approach and targeted pulmonary vascular therapy.

KEYWORDS: Caesarean section, iloprost, local anaesthesia, pregnancy, pulmonary hypertension

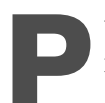

ulmonary arterial hypertension $(\mathrm{PAH})$ is a rare condition previously associated with a progressive course and few treatment options. Since the 1980s, new therapies have been developed which have improved patients symptoms and survival.

Idiopathic pulmonary arterial hypertension (IPAH) is a rare condition with an incidence of one to two cases per million per year [1]. Patients with severe IPAH have a 5 -yr survival of $27 \%$ with supportive treatment, increased to $54 \%$ with certain targeted pulmonary vascular therapies [2].

With pulmonary hypertension $(\mathrm{PH})$, pregnancy is a high-risk condition and its pre-existence or gestational occurrence is associated with a 30 $56 \%$ risk of maternal death [3]. Patients are counselled to avoid pregnancy or are offered an early interruption in its eventuality. However, PAH may only become apparent during pregnancy, or patients may decide to continue with their pregnancy even with this advice. Targeted pulmonary vasodilator therapy provides an option and a potential treatment dilemma for clinicians.

There have been reports on the utility of vasodilator therapy in the peri-partum period, but little published literature on the use of these drugs during early pregnancy.

The current study describes the early initiation of pulmonary vasodilator therapy using nebulised iloprost (a prostacyclin analogue) in a series of patients with PAH in pregnancy.

\section{METHODS}

A retrospective review of patients with $\mathrm{PH}$ treated at the Pulmonary Vascular Disease Unit, Sheffield, UK since July 2001 identified four individuals becoming pregnant who decided to continue their pregnancies despite physician

\section{AFFILIATIONS}

*Sheffield Pulmonary Vascular

Disease Unit,

\#Dept of Obstetrics and

Gynaecology,

"Dept of Anaesthesia and Critical

Care, and

+Division of Genomic Medicine,

University of Sheffield, Royal

Hallamshire Hospital, Sheffield, and

${ }^{\text {s}}$ Dept of Obstetrics and Gynaecology, and

${ }^{f}$ Leicester Royal Infirmary,

Congenital Heart Service, Glenfield

Hospital, Leicester, UK.

CORRESPONDENCE

D.G. Kiely

Sheffield Pulmonary Vascular

Disease Unit

Royal Hallamshire Hospital

Sheffield Teaching Hospitals NHS

Foundation Trust

Glossop Road

Sheffield

S10 2JF

UK

Fax: 441142711718

E-mail: david.kiely@sth.nhs.uk

Received:

November 092004

Accepted after revision:

March 282005

European Respiratory Journal

Print ISSN 0903-1936

Online ISSN 1399-3003 
advice. Three of these patients were treated with nebulised iloprost and are included in this report. The fourth presented with $\mathrm{PAH}$ at 24 weeks of gestation, with a right heart catheter at 26 weeks demonstrating: mean pulmonary artery pressure (mPAP) $33 \mathrm{mmHg}$; cardiac index $(\mathrm{CI}) 4.5 \mathrm{~L} \cdot \mathrm{min} \cdot \mathrm{m}^{-2}$; right atrial (RA) pressure $13 \mathrm{mmHg}$; mixed venous oxygen saturations $\left(M V, \mathrm{O}_{2}\right) 67 \%$; and total pulmonary vascular resistance (TPR) 377 dynes $\cdot \mathrm{s} \cdot \mathrm{cm}^{-5}$. A subsequent mediastinoscopy confirmed lymphoma. She delivered by caesarean section at 28 weeks, under local anaesthetic to allow the initiation of chemotherapy. The patient clinically deteriorated following the birth and repeat cardiac catheter demonstrated progressive disease despite the institution of chemotherapy $(\mathrm{mPAP}=$ $47 \mathrm{mmHg}, \quad \mathrm{CI}=2.4 \mathrm{~L} \cdot \mathrm{min} \cdot \mathrm{m}^{-2}$, RA pressure $=4 \mathrm{mmHg}$, $M V, \mathrm{O}_{2}=55 \%, \mathrm{TPR}=1,016 \mathrm{dynes} \cdot \mathrm{s} \cdot \mathrm{cm}^{-5}$ and pulmonary vascular resistance 865 dynes $\cdot s \cdot \mathrm{cm}^{-5}$ ). The patient was treated with nebulised iloprost but died as a result of neutropenic sepsis following chemotherapy 5 months after childbirth. The patient was treated with nebulised iloprost after (not during) pregnancy and was, therefore, excluded from this report.

No other individuals chose to continue their pregnancies and there was no pregnancy-related mortality. Another patient did become pregnant in this time period, but opted for termination of pregnancy. $\mathrm{PH}$ was defined as $\mathrm{mPAP} \geqslant 25 \mathrm{mmHg}$ at rest.

Iloprost was delivered via an ultrasonic nebuliser (Multisonic; Schill Medical, Probstzella, Germany) with dose escalation from 3-20 $\mu \mathrm{g}$ (highest tolerated dose), and made up with normal saline to a volume of $3 \mathrm{~mL}$ per nebulisation, seven times per day.

Cases 1 and 3 were delivered at the Royal Hallamshire Hospital, Sheffield, UK with case 2 being delivered at the Glenfield Hospital, Leicester, UK.

\section{CASE 1}

\section{Background}

A 23 yr-old female had been diagnosed with IPAH 3 yrs earlier after presenting with blackouts. Two years prior to this she completed a pregnancy (complicated by pre-eclampsia) with a caesarean section at 32 weeks of gestation.

The patient was clinically stable (New York Heart Association (NYHA) II) on treatment with bosentan (orally active endothelin receptor antagonist), having discontinued i.v. therapy after recurrent Hickman line infections and subcutaneous treprostinil due to site pain. She had been advised against pregnancy (due to the health risks it posed and the possible teratogenicity of bosentan), and had also received contraceptive advice from a gynaecologist.

\section{Pregnancy}

The patient returned to the clinic 6 weeks pregnant, clinically stable in NYHA functional class II with an incremental shuttle walking test distance (ISWT) of $330 \mathrm{~m}$. Physical examination demonstrated a pulse rate of 76 beats $\cdot \mathrm{min}^{-1}$, blood pressure $110 / 60 \mathrm{mmHg}$. Oxygen saturations were 95\% breathing room air. There was elevation of the jugular venous pressure (JVP), a right ventricular heave with a systolic murmur and a loud, palpable pulmonary component of the second heart sound. There was no peripheral oedema. Electrocardiograph (ECG) showed sinus rhythm with an axis of $70^{\circ}$ and a rate of 71 beats $\cdot \min ^{-1}$ with $\mathrm{T}$-wave inversion leads $\mathrm{V} 1-\mathrm{V} 3$. Transthoracic echocardiography (TTE) showed normal chamber sizes, estimating systolic pulmonary artery pressure (sPAP) at $50 \mathrm{mmHg}$.

Bosentan and warfarin were immediately stopped due to teratogenic potential. Low molecular weight heparin (LMWH) and nebulised iloprost were established at 8 weeks of gestation. The patient was reviewed at four weekly intervals with noninvasive investigations and remained stable with an ISWT distance of $320 \mathrm{~m}$ at 22 weeks of gestation.

At 24 weeks, the patient developed increased dyspnoea and cough, at which time she was admitted to hospital. There was no change in ECG, chest radiograph or echocardiogram.

At 24 weeks and 4 days, she experienced contractions and the obstetric team diagnosed threatened labour. She was treated with analgesics (diamorphine) and corticosteroids to aid maturation of the foetal lungs; contractions stopped and a cardiotocograph showed no evidence of foetal distress.

Despite intensifying treatment by increasing the nebulised iloprost to $8-9$ times q.d. (20 $\mu \mathrm{g}$ per nebulisation) there was deterioration in the control of PAH. At 25 weeks the patient collapsed suffering a cardiorespiratory arrest. After successful resuscitation (cardiopulmonary resuscitation lasting $\sim 1 \mathrm{~min}$ ) she was converted to i.v. iloprost (escalated to a dose of $2.2 \mathrm{ng} \cdot \mathrm{kg} \cdot \mathrm{min}^{-1}$ ) with haemodynamic monitoring via pulmonary artery catheter. After a period of 5 days, under local anaesthetic (an epidural was sited in the space between the third and forth lumbar vertebrae, L3/4, with $2.5 \mathrm{mg}$ diamorphine, $20 \mathrm{~mL} 0.5 \%$ bupivacaine in incremental doses) a lower segment caesarean section together with bilateral tubal ligation was performed. Due to failure of the uterus to contract (despite bimanual compression) syntocinon was given. After an initial test dose of 0.5 IU there was an increase in heart rate (from 84112 beats $\cdot \mathrm{min}^{-1}$ ) and $\mathrm{mPAP}$ (from $35-40 \mathrm{mmHg}$ ) with a fall in cardiac output (from 6.3-5.5 L. $\mathrm{min}^{-1}$ ), an effect less marked with repeated doses and with no adverse clinical sequelae. Total blood loss was $300 \mathrm{~mL}^{-1}$. The patient delivered a male infant $(0.65 \mathrm{~kg}$; American Pediatric Gross Assessment Record (APGAR) scores: 8 at $1 \mathrm{~min}$ and 9 at $5 \mathrm{~min}$ ), who was ventilated and transferred to the neonatal intensive care unit (ICU) for further care. Haemodynamic data for this period is recorded in table 1 , showing that severe $\mathrm{PAH}$ improved with i.v. iloprost.

\section{Post-delivery course}

The patient was monitored in the ICU. A two-unit blood transfusion was required due to anaemia. Maintaining fluid balance was difficult, and the patient had clinical evidence of fluid overload. Anticoagulation was recommenced $48 \mathrm{~h}$ postoperatively, initially with i.v. heparin, then LMWH and warfarin until the international normalised ratio was $>2$ for $48 \mathrm{~h}$. Central venous catheters were removed $72 \mathrm{~h}$ after delivery. At this time bosentan was restarted and i.v. iloprost was continued for 8 days, at which point it was converted back to the nebulised route. The patient stopped nebulised iloprost 1 month later and continued on bosentan and warfarin.

A period of 16 months later, the patient remains on bosentan and warfarin, in NYHA class II and can walk $430 \mathrm{~m}$ on ISWT. Her son is at home and is progressing well. 


\begin{tabular}{|c|c|c|c|c|c|c|c|c|}
\hline NYHA & $\|$ & $\|$ & $\|$ & $\|-I I I$ & IV & IV & IV & $\|$ \\
\hline ISWT m & 450 & 360 & 330 & 320 & & & & 430 \\
\hline TTE sPAP $\mathrm{mmHg}$ & \# & 50 & 50 & 59 & & & & 50 \\
\hline RA mmHg & 1 & & & & 18 & 3 & 1 & \\
\hline CO L. $\min ^{-1}$ & 5.8 & & & & 4.3 & 6.3 & 6 & \\
\hline $\mathrm{CI} L \cdot \min \cdot \mathrm{m}^{-2}$ & 3.7 & & & & 2.7 & 3.9 & 3.7 & \\
\hline PVR dynes $\cdot \mathbf{s} \cdot \mathrm{cm}^{-5}$ & 497 & & & & & & & \\
\hline TPR dynes $\cdot \mathbf{s} \cdot \mathrm{cm}^{-5}$ & 524 & & & & 1265 & 533 & 451 & \\
\hline$M V, \mathrm{O}_{2} \%$ & 68 & & & & & & & \\
\hline
\end{tabular}

NYHA; New York Heart Association; ISWT: incremental shuttle walking test distance; TTE sPAP: transthoracic echocardiography systolic pulmonary artery pressure; mPAP: mean pulmonary artery pressure; RA: right atrial; CO: cardiac output; Cl: cardiac index; PVR: pulmonary vascular resistance; TPR: total pulmonary resistance; $M V, \mathrm{O}_{2}$ : mixed venous oxygen saturations. ${ }^{\#}$ : trivial tricuspid regurgitation, estimate of sPAP not possible.

\section{CASE 2}

\section{Background}

A 29-yr-old female with two sisters that had died from $\mathrm{PH}$ (one following childbirth) had developed transient $\mathrm{PH}$ in a previous pregnancy with $\mathrm{PAP}=70 / 30 \mathrm{mmHg}$. In that pregnancy, a caesarean section was performed at 35 weeks due to increasing breathlessness. A diagnosis of familial PAH (FPAH) was made. Interestingly, during follow-up there was an early improvement in haemodynamics into the post-partum period with subsequent noninvasive assessments suggesting resolution of PAH. In view of her family history, genetic testing was carried out with particular reference to the bone morphogenetic protein receptor (BMPR)-II gene (known to be associated with FPAH). These studies demonstrated that although a recognised mutation was not present, the patient had the same BMPR-II haplotype as one of her sisters who had died of PAH.

She was strongly advised against pregnancy, but expressed a wish to conceive again.

\section{Pregnancy}

The patient subsequently presented with a confirmed pregnancy at 10 weeks of gestation and no increase in breathlessness. She was clinically stable and on examination there was a loud pulmonary component of the second heart sound. ECG was normal and TTE estimated sPAP at $45 \mathrm{mmHg}$. NYHA functional class was II with an ISWT distance of $450 \mathrm{~m}$. Due to the transient nature of $\mathrm{PAH}$ during her previous pregnancy, a repeat cardiac catheter was performed, which demonstrated a PAP of $60 / 30 \mathrm{mmHg}$ with $\mathrm{mPAP}=40 \mathrm{mmHg}$, RA pressure $=7 \mathrm{mmHg}$ and pulmonary capillary wedge pressure of $14 \mathrm{mmHg}$.

She commenced LMWH and nebulised iloprost at 19 weeks of gestation in view of the problems with progressive symptoms during her previous pregnancy. A dose of $10 \mu \mathrm{g}$ per nebulisation, seven times q.d., was the maximum tolerated due to headaches.
The patient remained clinically stable in NYHA class II-III until the premature onset of labour at 36 weeks.

Given her previous obstetric and family history it was decided to deliver her operatively and she was transferred to her local cardiothoracic unit for urgent caesarean section and elective sterilisation. A pulmonary artery catheter was inserted prior to siting her epidural $(15 \mathrm{~mL}$ mixture of $0.1 \%$ bupivicaine and fentanyl $\left.2 \mathrm{mg} \cdot \mathrm{mL}^{-1}\right)$. A lower segment caesarean section was performed (with bilateral partial salpingectomies) with the delivery of a male infant ( $2.8 \mathrm{~kg}$; APGAR 9 at 1 and $5 \mathrm{~min})$. The placenta was expelled spontaneously, syntocinon was not used and blood loss was minimised (total $400 \mathrm{~mL}$ ) via combination of careful surgical technique and bimanual compression of the uterus.

\section{Post-delivery course}

The patient was monitored on cardiac intensive care. Following the procedure her central venous pressure was $7 \mathrm{mmHg}$ and PAP $=65 / 30$. The Swan Ganz catheter was removed after $72 \mathrm{~h}$ and the patient transferred back to the delivery suite. Nebulised iloprost was stopped 1 month later with the patient in NYHA functional class II. LMWH was continued for 6 weeks post-natally. Subsequent echocardiograms show no evidence of $\mathrm{PAH}$, but pulmonary function testing demonstrated a persistently low transfer factor. The patient remains well 18 months following childbirth in NYHA class II with an ISWT distance of $440 \mathrm{~m}$ (table 2). To date, she has declined an invitation for repeat cardiac catheterisation and has a healthy son.

\section{CASE 3}

\section{Background}

A 24-yr-old female with three previous pregnancies had a heart murmur detected toward the end of the last pregnancy. She was diagnosed with an ostium secundum atrial septal defect with an echocardiogram estimate of sPAP of $50 \mathrm{mmHg}$. The patient had two previous full-term deliveries via the vaginal route and a stillbirth at 20 weeks. There was a history 
TABLE 2 Summary of recorded physiology for case 2

\begin{tabular}{lcccc} 
& $\begin{array}{c}\text { Previous } \\
\text { pregnancy }\end{array}$ & $\begin{array}{c}\mathbf{1 0} \text { weeks of } \\
\text { gestation }\end{array}$ & $\begin{array}{c}\text { Post-delivery } \\
\mathbf{1 8} \text { month } \\
\text { post-partum }\end{array}$ \\
\hline NYHA & $\|$ & $\|$ & III & $\|$ \\
ISWT $\mathbf{m}$ & & 450 & & 440 \\
TTE sPAP $\mathbf{m m H g}$ & & 45 & & 35 \\
PAP $\mathbf{m m H g}$ & $70 / 30$ & $60 / 30$ & $65 / 30$ & \\
mPAP $\mathbf{m m H g}$ & 48 & 40 & & \\
RA $\mathbf{m m H g}$ & 58 & 7 & 7 & \\
$\mathbf{M} \boldsymbol{N}, \mathrm{O}_{2} \%$ & 74 & 76 & & \\
\hline
\end{tabular}

NYHA; New York Heart Association; ISWT: incremental shuttle walking test distance; TTE SPAP: transthoracic echocardiography systolic pulmonary artery pressure; PAP: pulmonary artery pressure; mPAP: mean pulmonary artery pressure; RA: right atrial; $M V, \mathrm{O}_{2}$ : mixed venous oxygen saturations.

of increasing dyspnoea during her second and third pregnancies. She had been referred for further investigation but presented pregnant, 16 months following her previous delivery.

\section{Pregnancy}

At 17 weeks of gestation, NYHA functional class was II-III. JVP was elevated, and there was a right ventricular heave with systolic and early diastolic murmurs and pre-cordial thrill. She had no peripheral oedema. An ECG demonstrated sinus rhythm with a heart rate of 75 beats $\mathrm{min}^{-1}$, an axis of $100^{\circ}$ and ST segment changes throughout the chest leads. TTE estimated sPAP was $75-80 \mathrm{mmHg}$ and an ISWT distance of $140 \mathrm{~m}$ was achieved. In view of the patients symptomatic decline in previous pregnancies nebulised iloprost commenced, with a dose of $15 \mu \mathrm{g}$ per nebulisation, seven times daily. She was reviewed every 4 weeks with noninvasive investigations until 28 weeks of gestation after which she was seen weekly.

The initiation of treatment brought a symptomatic improvement and her walking distance increased to $190 \mathrm{~m}$.

At 34 weeks gestation abdominal pain and bleeding occurred and ultrasound examination revealed an anterior grade IV placenta praevia. Lower segment caesarean section was performed at 35 weeks under local anaesthesia, consisting of a lumbar epidural $2.5 \mathrm{mg}$ diamorphine and $15 \mathrm{~mL}$ of $0.5 \%$ bupivocaine in incremental doses to reach a block to touch $\mathrm{T} 4$ bilaterally; resulting in the birth of a female infant $(2.16 \mathrm{~kg}$; APGAR scores 9 at $1 \mathrm{~min}$ and 10 at 5 and $10 \mathrm{~min}$ ). This was complicated by intra-operative bleeding requiring a two-unit blood transfusion. Syntocinon was given with no adverse haemodynamic effects.

\section{Post-delivery course}

Due to ongoing bleeding the patient had to return to theatre (with epidural top up of $10 \mathrm{~mL} 0.375 \%$ bupivacaine) for the insertion of an intra-uterine balloon, which was successful. Post-operatively she developed lobar pneumonia that responded to standard antibiotic therapy. Two weeks postdelivery mother and infant were discharged well from hospital. The patient had a progestogen only contraceptive implanted. At 12 weeks post-delivery she was clinically stable in NYHA class II, with an ISWT distance of $180 \mathrm{~m}$ (table 3). Her daughter is currently well.

\section{DISCUSSION}

Pregnancy and PAH are undoubtedly associated with a high risk of maternal death. Current practice is to advise against pregnancy giving appropriate contraceptive recommendations and, if necessary, termination of pregnancy. Some patients opt to continue with their pregnancies in spite of this advice and a successful outcome is possible, as reported in the previous cases. Reviews of pregnancy in PAH report maternal mortality at $30-56 \%$ [3] and, interestingly, this is particularly high in the early post-partum period. However, this largely represents data prior to the advent of targeted pulmonary vascular therapies.

Recently there have been reports of successful pregnancies using inhaled pulmonary vasodilators $[4]$, i.v. therapy $[5,6]$ or a combination of the two routes of administration at different stages of pregnancy and the post-partum period [7]. These reports concern the use of pulmonary vasodilators exclusively in the third trimester. As well as successful reports, there are cases where maternal death cannot be avoided even with intensive specialist treatment $[5,8]$. These give a stark reminder that although newer therapies may present an option, they do not represent the answer to the difficulty of $\mathrm{PAH}$ in pregnancy.

The reported cases demonstrate the highly variable presentation and natural history of PAH and its progress in pregnancy (table 4). Two patients presented within the first trimester and the other early in the second, with no increase in breathlessness.

The decision to commence iloprost in previously untreated patients was based on worsening breathlessness through previous pregnancies. In the patient receiving bosentan it was felt necessary to continue targeted pulmonary vascular treatment, due to the unstable nature of her disease prior to pulmonary vascular therapy.

The introduction of inhaled iloprost (as early as the eighth week of gestation) was tolerated without adverse event and all

\begin{tabular}{lccccc|} 
TABLE 3 & Summary of recorded physiology for Case 3 \\
\cline { 2 - 6 } & $\mathbf{5}$ & $\mathbf{2 6}$ & $\mathbf{3 1}$ & $\mathbf{3 4}$ & $\mathbf{1 2}^{\#}$ \\
\cline { 2 - 6 } & 17 & Gestation weeks \\
NYHA & II-III & 11 II & IIII & II-III & ॥ \\
ISWT m & 140 & 190 & 170 & 190 & 180 \\
TTE sPAP $\mathbf{m m H g}$ & 75 & 80 & 70 & 65 \\
\hline
\end{tabular}

NYHA; New York Heart Association; ISWT: incremental shuttle walking test distance; TTE sPAP: transthoracic echocardiography systolic pulmonary artery pressure. ${ }^{\#}$ : post-partum. 


\begin{tabular}{|c|c|c|c|}
\hline & Case 1 & Case 2 & Case 3 \\
\hline Iloprost started weeks of gestation & 8 & 19 & 18 \\
\hline Dose achieved & $20 \mu \mathrm{g} 7$ daily doses & $10 \mu \mathrm{g} 7$ daily doses & $15 \mu \mathrm{g} 7$ daily doses \\
\hline Anticoagulation during pregnancy & Full dose LMWH & Full dose LMWH & None \\
\hline Precipitant & Increased dyspnoea & Premature labour & Abdominal pain and PV bleeding \\
\hline Delivery weeks of gestation and days & $25+5$ & 36 & 35 \\
\hline Mode & Caesarean section+epidural & Caesarean section+epidural & Caesarean section+epidural \\
\hline Follow-up & 16 months & 18 months & 12 weeks \\
\hline Treatment at follow-up & Bosentan & None & Nebulised iloprost \\
\hline
\end{tabular}

LMWH: low molecular weight heparin; PV: per vagina

infants were free from congenital birth defects. Of particular note was case 1 where conception occurred on bosentan treatment. This is one of only three completed pregnancies worldwide having been conceived with concurrent maternal use of bosentan [9], which has been shown to have teratogenic potential in animals.

In one case, the route of iloprost administration was switched to i.v. delivery due to symptom progression and this resulted in an immediate clinical improvement.

In all cases, delivery was operative with regional anaesthetic. This was performed without haemodynamic compromise or adverse effect on PAH. Case 3, involving a placenta praevia, did result in significant intra-operative and post-operative blood loss, but this was dealt with swiftly by blood replacement and operative means.

The optimum route of delivery is uncertain in PAH. In normal labour there is an increase in cardiac output by $12 \%$ above pre-labour levels and this has increased by $34 \%$ at full dilatation of the cervix [10]. Normal vaginal delivery, therefore, places an extra burden on the heart. However, operative delivery has been associated with higher maternal mortality in patients with PAH [3]. The reasons for this are not clear. Patients in whom vaginal delivery was chosen may have been tolerating their pregnancy particularly well. Those having operative interventions could have done so because of a worsening in their clinical condition or an obstetric emergency, thereby selecting a higher risk group. In all three patients operative delivery was preferred for a number of different reasons.

In all three patients the use of iloprost seems to have been associated with a better outcome than would have been expected, and was also associated with a symptomatic improvement in cases 2 and 3. The potential for iloprost use raises further questions, such as the optimal timing of treatment initiation, route of administration and dose used. In these matters, the individuals' progress in previous pregnancies acted as a guide along with their symptoms in the current pregnancy. In the absence of published evidence in pregnancy, the current authors were keen to avoid iatrogenic problems associated with drug use. In view of the relative clinical stability of the patients in NYHA classes II and III, the decision was made to use nebulised therapy. Nebulised iloprost is approved in Europe for the treatment of IPAH in NYHA class III. It has been demonstrated to be an effective treatment for PAH [11], and avoids the potential complications of i.v. lines, such as infections that can result in life-threatening sepsis. However, concerns exist with the use of nebulised iloprost due to its short half-life and the potential for rebound PAH between nebulisations. In patients with severe disease (NYHA class IV), i.v. administration of prostaglandin is often advocated as first-line therapy. Nebulised iloprost was initiated and continued where there was a favourable response, converting to i.v. drug if there was clinical deterioration as in case 1 . Based on the authors' limited experience, a pregnant patient deteriorating on nebulised treatment should be immediately converted to i.v. therapy (unless any specific problems are anticipated). In case 1, there is no doubt this was life saving. In addition, in patients in NYHA class IV at presentation the authors would advocate i.v. treatment as first-line therapy. Currently, epoprostenol remains the only licensed i.v. therapy in Europe for the treatment of PAH. It is also the only i.v. prostaglandin having shown a survival benefit in PAH in randomised controlled trials. Iloprost (i.v.) is licensed for the treatment of PAH elsewhere and is the most commonly used i.v. therapy for PAH in the UK, due to its stability and ease of use. The present authors have extensive local clinical experience with i.v. iloprost, which is why it used in preference to i.v. epoprostenol in case 1 .

Anticoagulation is another issue, and the current authors have had no adverse events or problems with the use of full dose LMWH during pregnancy, or the reintroduction of warfarin in the post-partum period. In theory, prostaglandins may worsen bleeding in the peri-partum period due to their anti-platelet effect, but it has been shown that platelet aggregation tests are unchanged when performed before and after the use of inhaled iloprost (to cover a caesarean delivery [4]), and they were comparable to the hypercoagulable state usually seen near term. In patients who are unwell, a switch would be made from full dose to prophylactic anticoagulation and withheld for $24 \mathrm{~h}$ prior to the expected time of delivery to reduce haemorrhagic risk with local anaesthetic and other procedures. 
The three patients in this study saw the physician responsible for the care of their pulmonary vascular disease (and with case 2 the local cardiologist) and their obstetrician regularly and frequently throughout their pregnancies. They were also in close contact with specialist nurses for support, advice and to report any change in their symptoms. Key to the process was the early involvement of anaesthetic and critical care teams with advanced preparation and clear planning for the peripartum period.

\section{CONCLUSION}

Patients with pulmonary arterial hypertension should be advised against pregnancy with clear contraceptive advice and, if needed, early interruption of pregnancy. When patients who are fully informed and understand the risks of doing so chose to continue their pregnancies, early treatment with first-line nebulised iloprost, and if necessary, second-line intravenous prostaglandin, represents a realistic option and may improve the chances of maternal survival. With timely admission to hospital and close cooperation between a multidisciplinary team, a successful outcome for mother and foetus is possible.

\section{REFERENCES}

1 Rich S, Dantzker DR, Ayres SM, et al. Primary Pulmonary Hypertension. A national prospective study. Ann Intern Med 1987; 107: 216-223.

2 Rubin LJ, Mendoza J, Hood M, et al. Treatment of pulmonary hypertension with continuous intravenous prostacyclin (epoprostenol). Results of a randomised trial. Ann Intern Med 1990; 112: 485-491.
3 Weiss BM, Zemp L, Seifert B, Hess OM. Outcome of pulmonary vascular disease in pregnancy: A systematic overview from 1978 through 1996. J Am Coll Cardiol 1998; 31: $1650-1657$.

4 Weiss BM, Maggiorini M, Jenni R, et al. Pregnant patient with primary pulmonary hypertension: Inhaled pulmonary vasodilators and epidural anaethesia for caesarean delivery. Anaesthesiology 2000; 92: 1191-1194.

5 Easterling TR, Ralph DD, Scmucker BC. Pulmonary hypertension in pregnancy: Treatment with pulmonary vasodilators. Obstet Gynaecol 1999; 93: 494-498.

6 Stewart R, Tuazon D, Olson G, Duarte AG. Pregnancy and primary pulmonary hypertension. Successful outcome with epoprostenol therapy. Chest 2001; 119: 973-975.

7 Hill LL, De Wet CJ, Jacobsohn E, Leighton BL, Tymkew H. Peripartum substitution of inhaled for intravenous prostacyclin in a patient with primary pulmonary hypertension. Anaesthesiology 2004; 100 1603-1605.

8 Monnery L, Nanson J, Charlton G. Primary pulmonary hypertension in pregnancy; a role for novel vasodilators. Br J Anaesth 2001; 87: 295-298.

9 3rd TRAX PMS Data Report to CPMP for the period from 24 May 2002 to 19 February 2003 (Actelion Pharmaceuticals, data on file).

10 Hunter S, Robson SC. Adaption of the maternal heart in pregnancy. Br Heart J 1992; 68: 540-543.

11 Olschewski H, Simonneau G, Galie N, et al. Aerosolized Iloprost Randomized Study Group. Inhaled Iloprost for severe pulmonary hypertension. N Engl J Med 2002; 347: 322-329. 